\title{
IMAGEN DEL MES
}

\section{DIVERTÍCULO DEMECKEL ASOCIADO A OBSTRUCCIÓN INTESTINAL SECUNDARIA A INVAGINACIÓN}

\section{Meckel's diverticulum associated to bowel obstruction caused by intussusception}

Torrecillas Cabrera MM, Moya Sánchez E, Fernández Navarro L HOSPITAL UNIVERSITARIO CLÍNICO SAN CECILIO. GRANADA.

\section{Resumen}

La invaginación intestinal en adultos jóvenes constituye una entidad infrecuente, y en pocos casos da lugar a una obstrucción, además suelen ser secundarias a otra patología subyacente.

Se describe el caso de un varón de 18 años que acude a urgencias con cuadro de abdomen agudo, y es diagnosticado mediante radiografía simple y ecografía de obstrucción intestinal secundaria a invaginación de ileo-ileal, con divertículo de Meckel asociado.

Palabras clave: divertículo de Meckel, invaginación, obstrucción intestinal, ecografía, complicaciones.

\section{Abstract}

Intussusception in young adults is a rare entity, and in few cases it can lead to an intestinal obstruction, in addition they are usually secondary to another underlying pathology.

\section{CORRESPONDENCIA}

Maria del Mar Torrecillas Cabrera/ 137mmar@gmail.com Hospital Universitario Clínico San Cecilio - 18016. Granada Fecha de envío: 07/06/2021 - Fecha de aceptación: 10/02/2022
We present the case of an 18-years-old male who was admitted to the Emergency Department due to acute abdomen, and was diagnosed with intestinal obstruction secondary to ileo-ileal intussusception, associated with a Meckel's diverticulum, by $\mathrm{x}$-ray and ultrasound.

Keywords: Meckel's diverticulum, intussusception, intestinal obstruction, ultrasound, complications.

\section{Introducción}

El divertículo de Meckel constituye la anomalía congénita gastrointestinal más frecuente, su clínica deriva de sus complicaciones siendo la obstrucción intestinal la complicación más frecuente en adultos, entre cuyas causas destacan: inversión del divertículo, invaginación intestinal, vólvulo, hernia interna o externa o neoplasia. Esta entidad constituye un reto diagnóstico siendo en muchas ocasiones un diagnóstico incidental, por lo que es necesario estar familiarizado con su aspecto radiológico normal así como el de sus posibles complicaciones. 


\section{Caso clínico}

Varón de 18 años sin antecedentes de interés, que acude a urgencias por dolor abdominal y vómitos de dos días de evolución, con última deposición hace más de 24 horas.

A la exploración impresiona de masa en flanco derecho que se corresponde con la región más dolorosa.

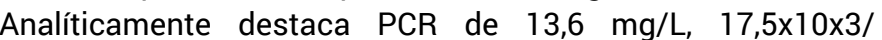
microL leucocitos, con $79 \%$ de neutrófilos, y alteración de los parámetros de la coagulación.

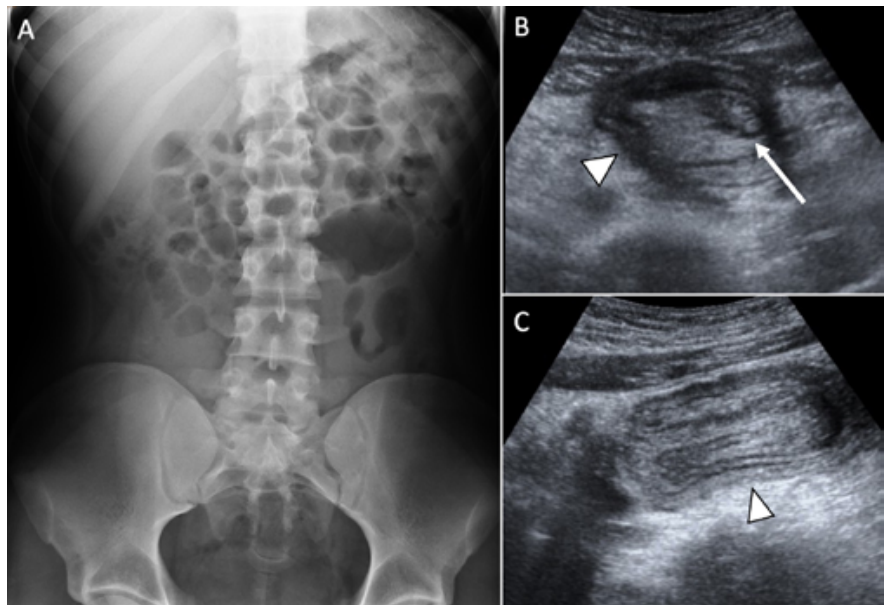

FIGURA 1

A. RADIOGRAFÍA AP DE ABDOMEN DONDE SE OBSERVA DILATACIÓN DE ASAS DE DELGADO HASTA ÍLEON PRETERMINAL. B Y C. ECOGRAFÍA CON IMAGEN SUGERENTE DE INVAGINACIÓN ILEO-ILEAL (CABEZAS DE FLECHA), EN CUYO INTERIOR SE APRECIA ADEMÁS DE HIPERECOGENICIDAD DE LA GRASA MESENTÉRICA, UNA ESTRUCTURA TUBULAR OUE PUDIERA CORRESPONDER CON UN DIVERTÍCULO DE MECKEL (FLECHA GRUESA).

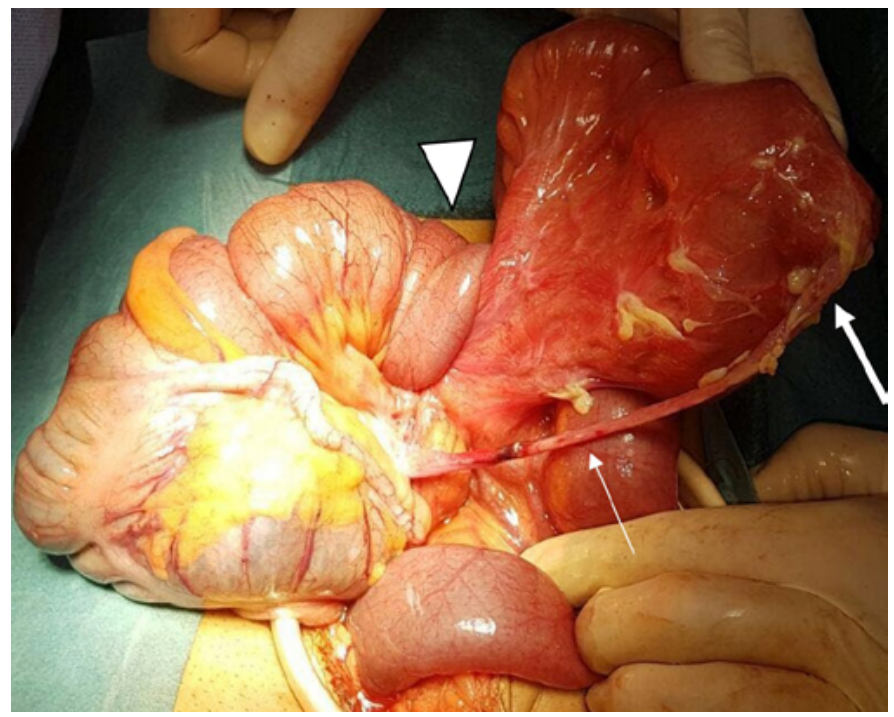

FIGURA 2

IMAGEN DE INTERVENCIÓN QUIRÚRGICA DONDE SE CONFIRMA LA INVAGINACIÓN ILEO-ILEAL (CABEZA DE FLECHA), CON UN DIVERTÍCULO DE MECKEL A UNOS 10 CM DE LA MISMA (FLECHA GRUESA), DEL CUAL PARTE UNA BRIDA HACIA LA RAÍZ DEL MESENTERIO (FLECHA).
Se realiza radiografía (Figura $1 \mathrm{~A}$ ) observando dilatación de asas de delgado hasta íleon preterminal, y ecografía (Figuras 1B y C) con imagen sugerente de invaginación ileo-ileal, en cuyo interior se aprecia probable divertículo de Meckel. En intervención quirúrgica urgente (Figura 2) se confirma invaginación, con un divertículo de Meckel a unos centímetros de la misma, con una brida hasta la raíz del mesenterio.

\section{Discusión}

El divertículo de Meckel constituye la anomalía congénita gastrointestinal más frecuente, se localiza en ileon distal ${ }^{1-4} y$ es resultado de una atrofia incompleta del conducto onfalomesentérico que en el embrión comunica el saco vitelino con el intestino ${ }^{2-4}$. Se trata de un verdadero divertículo ya que tiene todas las capas de la pared intestinal ${ }^{4}$.

Aproximadamente el $50 \%$ de los casos contienen mucosa heterotópica, más frecuentemente gástrica, aunque también se pueden encontrar tejidos como pancreático, mucosa yeyunal y duodenal entre otros ${ }^{3}$.

La incidencia es del $2 \%^{1-3}$,normalmente antes de los 2 años ${ }^{1,3}$,el $2 \%$ presentará complicaciones ${ }^{1}$ y es 2 veces más común en hombres ${ }^{3}$.

En estudios con bario, el divertículo de Meckel puede detectarse como una estructura tubular o sacular ciega que surge del borde antimesentérico del íleon terminal ${ }^{4}$.

En ecografía y en TC, suele aparecer como una estructura tubular ciega que surge de las asas ileales con una pared gruesa que consta de todas las capas de una pared intestinal normal ${ }^{4}$, sin embargo tanto la ecografía como la TC son poco útiles para la detección de un divertículo no complicado ${ }^{3}$

La mayoría de los divertículos de Meckel no producen síntomas, su clínica deriva de las complicaciones ${ }^{1-3}$. En niños la complicación más frecuente es el sangrado debido a la mayor prevalencia de mucosa gástrica ectópica ${ }^{3}$, en estos casos la gammagrafía con tecnecio 99 puede ser una herramienta útil para su detección ${ }^{3,4}$, mientras que en adultos predominan la obstrucción intestinal y la diverticulitis ${ }^{1,2}$.

Entre los mecanismos de obstrucción destacan la inversión diverticular provocando obstrucción luminal o invaginación intestinal ${ }^{2}$. Debe plantearse el diagnóstico diferencial en adultos jóvenes con obstrucción intestinal sin causa aparente ${ }^{1}$.

En TC el divertículo invertido se observa como un núcleo central de atenuación grasa rodeado por una zona de densidad de partes blandas. En caso de invaginación muestra anillos concéntricos alternados de atenuación grasa y tejidos blandos. 
En ecografía, el divertículo invertido tiene una apariencia de diana con hiperecogenicidad central por la grasa mesentérica, o una apariencia de doble diana cuando se visualiza toda la sección del intestino delgado que contiene el divertículo invertido ${ }^{2}$.

Por otro lado, la diverticulitis de Meckel produce una sintomatología similar a la apendicitis aguda, entre sus mecanismos etiopatogénicos destacan el efecto del ácido producido por la mucosa gástrica ectópica sobre la mucosa intestinal adyacente, o puede deberse a obstrucción del divertículo por enterolitos que se forman en su interior ${ }^{3}$.

El tratamiento en asintomáticos es controvertido ${ }^{3,4}$. La resección por laparoscopia es el tratamiento de elección en pacientes sintomáticos ${ }^{4}$. Además está indicada su resección si: cuello diverticular estrecho, masa palpable dentro del divertículo, banda fibrosa que comunique con el divertículo, y divertículo con vaso vitelino propio y sin mesenterio que predisponga a la encarcelación ${ }^{3}$.

El radiólogo debe estar familiarizado con el aspecto normal del divertículo y de sus posibles complicaciones para poder realizar un diagnóstico diferencial exhaustivo que oriente el tratamiento.

\section{Bibliografía}

1. Chatterjee $A$, Harmath $C$, Vendrami $C L$, et al.Reminiscing on Remnants: Imaging of Meckel Diverticulum and Its Complications in Adults. AJR Am J Roentgenol. 2017;209(5):287-296. doi: 10.2214/AJR.17.18088.

2. Levy $A D$, Hobbs CM. From the archives of the AFIP. Meckel diverticulum: radiologic features with pathologic Correlation. Radiographics. 200424(2):565-87. doi: 10.1148/rg.242035187.

3. Motta-Ramírez GA, Reyes-Méndez E, Campos-Torres J, et al. El divertículo de Meckel en adultos. Anales de Radiología México. 2015;14(1):20-30.

4. Kotha VK, Khandelwal A, Saboo SS, Shanbhogue AK, et al. Radiologist's perspective for the Meckel's diverticulum and its complications. $\mathrm{Br} \mathrm{J}$ Radiol. 2014;87(1037):20130743. 